\title{
Variations
}

Variations

Revue internationale de théorie critique

$22 \mid 2019$

Gorz, l'intempestif

\section{Lien valeur-nature dans la pensée d'André Gorz}

\section{Emanuele Leonardi}

\section{(2) OpenEdition}

Journals

Édition électronique

URL : http://journals.openedition.org/variations/1211

DOI : 10.4000/variations. 1211

ISSN : 1968-3960

\section{Éditeur}

Les amis de Variations

\section{Référence électronique}

Emanuele Leonardi, «Lien valeur-nature dans la pensée d'André Gorz », Variations [En ligne], 22 | 2019, mis en ligne le 04 mars 2019, consulté le 01 mai 2019. URL : http://journals.openedition.org/ variations/1211; DOI : 10.4000/variations. 1211

Ce document a été généré automatiquement le 1 mai 2019.

Les ami•e•s de Variations 


\title{
Lien valeur-nature dans la pensée d'André Gorz
}

\author{
Emanuele Leonardi
}

\section{Introduction ${ }^{1}$}

1 Mon intérêt pour la pensée de Gorz a toujours été 'instrumental' plutôt qu"interprétatif : dans le livre que j'ai récemment publié en italien (Leonardi, 2017a) il n'y a aucune reconstruction systématique de sa trajectoire socio-philosophique - la biographie de Willy Gianinazzi (2016) et la recherche de Françoise Gollain (2018) satisfont pleinement la nécessité d'un cadre exégétique solide. Ma tentative est plutôt d'utiliser l'œuvre de Gorz comme guide conceptuel pour réfléchir sur certaines questions étroitement liées à l'actualité: les passages 'gorziens' sont toujours mélangés avec des suggestions appartenant à d'autres traditions politiques et culturelles, d'une manière qui se justifie à partir de la relation entre le problème objet d'étude et la volonté de le comprendre adéquatement pour l'attaquer politiquement.

2 La question sur laquelle je me suis concentré ces dernières années est l'hégémonie de l' économie verte dans le domaine des politiques environnementales au niveau international. Par 'économie verte' j'entends la tentative capitaliste d'internaliser la limite environnementale pour la transformer d'obstacle à la valorisation en élément d'un nouveau cycle d'accumulation. En effet, il est incontestable que les élites mondiales misent sur le fait que la limite environnementale ne représente pas une contrainte au développement, mais une opportunité économique et un moteur de croissance sans précédent.

3 Considérons par exemple le récent Accord de Paris (2015), qui propose comme pierre angulaire économique de la politique climatique l'idée que c'est dans le marché que se trouve le salut de la planète. La commercialisation de la lutte contre le réchauffement climatique a été relancée, malgré l'insuffisance avérée de cette stratégie avec le Protocole de Kyoto. Sur ce point, le secrétaire d'État américain John Kerry, négociateur en chef à la COP 21 à Paris, n'a laissé aucune place à l'incompréhension : 
« Ici, à Paris, nous envoyons un message extraordinaire aux marchés, c'est-à-dire que 196 pays sont impliqués dans la lutte contre le changement climatique. Cela aidera le secteur privé à investir dans cette lutte, car il est maintenant clair qu'il existe un avenir dans cette voie de la durabilité » (dans McMahon, 2015).

Ce Consensus de Paris est généralisé - à l'exception, solitaire mais très importante, de Donald Trump - et tout axé sur la promotion de l'efficience économique et de l'innovation 'verte' comme facteurs simultanés de réduction des coûts et de préservation environnementale.

Il s'agit d'un parfait exemple de la façon dont le marché fonctionne - même en référence à la question écologique - comme lieu de véridiction, selon la formule proposée par Michel Foucault dans ses cours sur la biopolitique (Leonardi, 2017b).

6 C'est cette manière très particulière de comprendre la relation entre nature et valeur véhiculée par l'économie verte que représente l'objet spécifique sur lequel je vais essayer - avec l'aide de Gorz - de concentrer mon analyse critique. Dans cet article, l'intention n'est pas tant de falsifier les propositions par lesquelles cette connexion se présente, mais d'en esquisser une généalogie - de montrer comment elles ont émergé historiquement de manière à pouvoir les problématiser adéquatement. Dans ce contexte, l'écologie politique est un dispositif utile pour élargir la critique de l'économie politique de manière à y inclure la relation entre le capitalisme et l'environnement, c'est-à-dire à remettre en question le développement historique du lien valeur-nature.

7 Je commence par une notation préliminaire : l'économie verte est une question plus compliquée qu'il ne parait. Elle semble être frappée au coin du bon sens - qui ne voudrait pas obtenir, à la fois, protection de l'environnement et croissance économique ? - mais les conditions politico-épistémologiques qui la font émerger comme telle sont vraiment très récentes. En fait, dans les années Soixante et au début des années Soixante-dix - c'est à dire, dans la période de transition où la crise écologique explose en tant que problème proprement politique - l'idée que la détérioration des conditions écologiques pourraient être la force motrice d'un nouveau cycle d'accumulation capitaliste était tout simplement impensable. Bien sûr, il y avait ceux qui ont correctement mis en garde contre le risque d'une utilisation anti-ouvrière de l'écologie (Gorz même d'entre eux). Cependant, c'était une tentative de ne pas sous-estimer les capacités d'adaptation montrées par le capitalisme au cours de son développement historique, certainement pas une préfiguration de l'économie verte.

8 Elle émergera plus tard - vers la fin des années Quatre-vingt-dix - et après vingt ans de mise en œuvre, on peut affirmer qu'il s'agit d'une posture dogmatique dont la puissance sociale est extrême, indifférente à tout déni pratique. D'une part, elle est définie par sa nature de croyance exclusive et non basée sur l'analyse rationnelle du matériel empirique disponible; d'autre part, elle reflète uniquement une conception présentant le rôle salvateur du système de prix, en supposant que, bien que la crise écologique - et en particulier le changement climatique - soit une défaillance du marché (qui par le passé n'a pas été capable de l'internaliser en le reléguant essentiellement aux externalités), elle ne peut néanmoins être traité efficacement que sur la base d'une poursuite de la commercialisation. En d'autres termes: «donnez un prix à la nature et le problème disparaitra! ».

9 Avec une expression plus analytique: de nouveaux marchés exclusivement dédiés impliquent la production de nouvelles marchandises qui, à leur tour, sont le moteur d'une nouvelle vague d'accumulation primitive - enfin durable (en théorie). Cela fonctionne 
comme si l'on prescrivait à un patient un traitement proposant simplement des doses plus élevées de l'agent reconnu comme une cause directe de la maladie: le marché, en fait, agit contre le changement climatique dans le double rôle ambigu de bourreau comme coupable de négligence théorique et pratique - et de rédempteur - comme finalement capable de l'inclure correctement dans le système de prix.

Dans la tension entre objectifs environnementaux (subreptices) et moyens monétaires (réels) de l'économie verte, apparaît toujours plus clairement une création de valeur significative sous forme d'une rente nouvelle à côté des résultats écologiques nuls sinon négatifs. Cependant, quelles que soient les évaluations des résultats concrets des politiques dites 'vertes', il convient de noter tout de suite qu'ils ne sont ni faux, ni irrationnels: ils apparaîssent plutôt comme appropriés au régime d'accumulation contemporaine et, en même temps, fallacieux en ce qui concerne la typologie de travail qui produit de la valeur dans ce régime. La quasi-totalité des sujets participants sont perdants - dans une large majorité, les peuples dépossédés de leurs territoires, au Sud comme dans le Nord global ; peu de sujets sont gagnants - essentiellement des opérateurs financiers ; personne ne semble intéressé à se demander ce qui est arrivé au travail (ou à la force de travail) dans le lien valeur-nature qui soutient l'économie verte. Pour citer une expression très efficace d'Antonio Negri (2007 [1970]) - en l'appliquant, toutefois, dans un contexte différent de celui dans lequel il a été conçu - elle se présente comme « idéologie raisonnable $"^{2}$ de la tendance contemporaine du développement capitaliste, basée sur la mobilisation de plus en plus importante des activités reproductives/cognitive et sur la nouvelle centralité des marchés financiers dans le cadre de la gouvernance économique.

Voici donc la question de recherche qui organise la présentation : quelles transformations historiques ont créé les conditions par lesquelles une grande majorité des décideurs politiques trouvent raisonnable l'économie verte comme lien particulier entre la valeur et la nature ? Je vais essayer de répondre à travers trois mouvements 'gorziens' : mettant en évidence le caractère méthodologique de l'écologie politique pour situer le lien valeurnature qui était hégémonique jusqu'au Fordisme (c'est-à-dire celle proposée par l'économie politique classique) (\$1), analysant le choc pétrolier de 1973 comme un double crise (de suraccumulation et de reproduction) (\$2), et proposant quelques hypothèses sur la nouvelle configuration du lien valeur-nature dans le capitalisme contemporain (cognitive, financiarisée, basée sur la reproduction) pour discuter la question suivant : la crise écologique, est-elle compatible avec non seulement la survie, mais le développement, du système capitaliste ? (\$3).

Il me semble important de signaler immédiatement l'élément méthodologique sur lequel la réflexion gorzienne est greffée dans sa 'phase écologique' (environ 1971-1978). Voici le noyau fondamental de l'écologie politique tel que le conçoit André Gorz: la crise de la nature n'est pas extérieure à l'économie, à l'histoire, à la politique ; elle en est plutôt le visage extrême et le symptôme inévitable. Conformément à la tradition du matérialisme historique, la relation entre la nature et la société n'est pas donnée en termes abstraits, mais plutôt à partir du 'filtre' du mode de production et de ses transformations (Lavignotte, 2017).

14 On lit dans Écologie et liberté:

15 «L'écologie n'apparaît comme discipline séparée que lorsque l'activité économique détruit ou perturbe durablement le milieu ambiant et, de ce fait, compromet la poursuite 
de l'activité économique elle-même, ou en change sensiblement les conditions. L'écologie s'occupe des conditions que l'activité économique doit remplir et des limites externes qu'elle doit respecter pour ne pas provoquer des effet contraires à ses buts ou même incompatibles avec sa propre continuation » (Gorz, $1977: 22$ ).

En effet, Gorz est parmi les premiers à penser à la question environnementale dans sa non-autosuffisance, dans son impossibilité de s'expliquer par elle-même : elle ouvre une crise du productivisme occidental et du capitalisme industriel qui a une origine historique précise (c'est-à-dire la double crise - sociale et environnemental - apparue avec le premier choc pétrolier de 1973) et qui présente un enjeu de transformation sociale sans précédent.

fait, la dégradation des équilibres bio-sphériques ouvre un scénario fortement polarisé : à la tentation despotique doit faire face un projet social complexe combinant durabilité environnementale et autonomie aux niveaux individuel et collectif : « Il est [...] impossible de faire découler de l'écologie une morale [...] Le rejet du technofascisme ne procède pas d'une science des équilibres naturels mais d'une option politique et de civilisation » (Gorz, 1977 : 25-27).

En d'autres termes, le lien entre écologie et liberté n'est pas une donnée de nature - il n'est pas dans les choses : il faut le produire, le soigner, le défendre. Pour ce faire, la première étape est analytique : comment se configure le lien entre la valeur et la nature dans le capitalisme? Sur la base des réflexions de Ricardo, Marx a correctement noté comment la nature à laquelle se réfère le capital est immédiatement interne à ses mécanismes de valorisation. Cependant, c'est une interiorité particulière : d'une part, loin de se présenter comme transcendant par rapport à l'interaction entre les forces productives et les rapports sociaux de production, la nature est posée comme capital, comme mode spécifique de son existence ; d'autre part, elle est construite comme externe et passive dans une conception de la valeur en tant qu'agent auto-propulseur qui vise à son expansion à travers l'exploitation de sa source, c'est-à-dire le travail social abstrait (dans sa forme salarié).

19 Pour simplifier, ceci est le schéma qui vient de l'économie politique classique : le circuit de la valeur place la nature comme condition - infinie et gratuite à la fois au début du processus économique (matières premières) et à sa fin (élimination des déchets) - et en même temps place le travail abstrait comme facteur de ce processus lui-même. Marx ajoute à ce cadre sa critique, visant à contrebalancer la soi-disant 'dimension progressiste du capital' : dans le mode de production capitaliste, l'augmentation des niveaux de bienêtre social s'obtient uniquement au prix de l'exploitation des travailleurs et de la dégradation de l'environnement.

Ce raisonnement n'implique pas que le capitalisme soit destiné à briser les équilibres biosphériques (bien que les données empiriques que nous avons aujourd'hui suggèrent exactement cela). Il implique plutôt que le lien valeur-nature 'classique' - en tant que structure de pouvoir social constitutivement entropique - est à l'origine de la crise écologique, devenue politiquement perceptible dans les années 1970 (mais incubée depuis le 16ème siècle). De ce point de vue, le Fordisme doit donc être considéré comme le sommet du lien valeur-nature 'classique', c'est-à-dire comme un dispositif entropique orienté vers la croissance. En fait, considérant l'environnement infini et gratuit, ce lien d'une part perd la référence à ce que Gorz a appelé « norme du suffisant » et d'autre part, il a tendance à sur-utiliser pour garantir l'accumulation : 
«Sous l'angle écologique, l'accélération de la rotation du capital conduit à exclure tout ce qui diminue dans l'immédiat le profit. L'expansion continuelle de la production industrielle entraîne donc un pillage accéléré des ressources naturelles. Le besoin d'expansion illimitée du capital le conduit à chercher à abolir la nature et les ressources naturelles pour les remplacer par des produits fabriqués, vendus avec profit » (Gorz, 2008 [2005] : 72).

En d'autres termes, leur travail méta-industriel - pour reprendre la formule suggestive proposée par Ariel Salleh (2010) pour définir les pratiques nécessaires à la reproduction de la vie sociale - est ignoré par la non-rémunération ou, pire, nié comme un éco-facteur néguentropique et donc irréductible à la dimension extractive-industrielle.

\section{Le choc pétrolier de 1973 : une double crise (de suraccumulation et de reproduction)}

Toutefois, il convient de noter que, sous une forme pure, ce pacte n'a jamais été mis en œuvre parce que constamment traversée par une pluralité de luttes dont la poussée cumulative a mené - dans la période 1968-1973 - à la crise irréversible du Fordisme comme dispositif d'entropie. À cet égard, je crois que la contribution la plus importante d'André Gorz par rapport au lien valeur-nature est dans sa lecture originelle du premier choc pétrolier (1973), c'est-à-dire l'événement qui marque la transformation de la crise écologique mondiale d'un problème de gestion technique en une question spécifiquement politique. Jusqu'à Écologie et liberté (1977), le choc pétrolier avait été interprété de deux façons : d'abord, comme une crise des matières premières, une réaction plutôt agitée à la 'découverte' des limites physiques de la croissance qui avait été soulignée par le rapport du Club de Rome l'année précédente. Ensuite, comme une tentative d'utiliser une crise capitaliste sévère à la fois pour démêler la soi-disant rigidité ouvrière, c'est-à-dire la puissance acquise par le mouvement ouvrier, et pour désintégrer la force de la nouvelle subjectivité révolutionnaire émergée en 1968.

En général, les deux lignes d'interprétation ne se sont pas croisées, restant dans la plupart des cas indifférentes l'une à l'autre. De ce point de vue, la contribution de l'écologie politique de Gorz est vraiment remarquable: en fait, il élabore une théorie multiple mais cohérente de la crise capitaliste qui a explosé en 1973. D'une part, à partir d'une acceptation de l'indication marxienne de la baisse tendancielle du taux de profit c'est-à-dire l'impossibilité dans le long terme de remplacer la fonction valorisatrice du travail ouvrier par le travail mort cristallisé dans les machines - il décrit une situation de suraccumulation que le capital produit en recourant à certains facteurs de contre-tendance (en particulier obsolescence programmée des produits et création de besoins induits, c'est-à-dire prédéterminés par le capital). D'autre part, Gorz identifie une crise de reproduction due aux coûts croissants que le capital doit supporter pour régénérer l'environnement (jusqu'alors utilisé comme ressource 'infinie' et 'gratuite') pour pouvoir le polluer à nouveau - avec un augmentation des prix comme conséquence inévitable. On lit dans un passage particulièrement important :

«Ce qui importe pour le moment, c'est que cette fuite en avant [basée sur l'obsolescence programmée des produits et sur les besoins induits découplé de leur valeur d'usage] s'est terminée à l'occasion de la crise du pétrole. Celle-ci n'a pas causé la dépression économique; elle a déclenché et révélé une dépression qui couvait depuis plusieurs années. Surtout, elle a fait toucher du doigt le fait que le développement capitaliste avait provoqué des raretés absolues: en essayant de 
surmonter les obstacles économiques à la croissance, le développement capitaliste

avait fait naître des obstacles physiques » (Gorz, 1977 : 46-47). saison conflictuelle 1968-1973, il devient possible de poser la question suivante: le capitalisme avancé peut-il intégrer la crise écologique comme facteur de développement, ou est-il forcé de la considérer comme un pur coût, c'est-à-dire d'accepter une tension insoluble entre économie et environnement? Selon les termes de Gorz: «l'équilibre global, dont la non-croissance - sinon la décroissance - de la production matérielle est une condition, cet équilibre global est-il compatible avec la survie du système? » (Gorz, $1972 b$ : 300). 
eepond à cette question de différentes manières en fonction du moment historique. J'ai isolé trois réponse, qui me semblent fondamentales pour analyse les transformations du lien valeur-nature. En 1972, lors d'un débat au Nouvel Observateur sur le rapport du Club de Rome intitulé Halte à la croissance ?, la réponse est oui et l'analyse rien de moins que visionnaire :

« La non-croissance est contraire à la logique du système capitaliste et incompatible avec le fonctionnement du capitalisme tel que nous le connaissons; elle n'est pas nécessairement incompatible avec la survie du capitalisme sous une autre forme, pendant une période limitée, mais qui peut être longue [...] Grâce [au monopole de la dépollution et du recyclage] un nouveau cycle d'accumulation pourra s'amorcer, fondé sur la capitalisation de la Nature elle-même, sur la subsomption par le capital de la totalité des facteurs et des conditions permettant la vie sur terre » (Gorz, $1972 b$ : 301).

En 1977, dans Ecologie et liberté, Gorz s'aligne avec la croyance répandue que la réponse à la question devrait être un non et fournit une analyse marxiste de cette impossibilité du capitalisme à incorporer la crise écologique non seulement comme coût mais comme facteur du développement. En se référant aux opérations de recyclage des industries chimiques allemandes dans la vallée du Rhin, Gorz écrit :

« La nécessité d'un tel recyclage a un signification économique précise : elle révèle qu'il faut désormais reproduire ce qui, jusqu'ici, était abondant et gratuit. L'air et l'eau, notamment, doivent être traités comme des moyens de production parmi d'autres : il faut investir dans des installations de dépollution qui redonnent à l'air et à l'eau certaines de leur qualités primitives. La conséquence de cette nécessité est une augmentation supplémentaire de la composition organique du capital [...] Et à cette augmentation ne peut correspondre aucune augmentation de la production marchande : l'air et l'eau qu'un trust chimique recycle ou dépollue après usage ne peuvent être vendus. La tendance à la baisse du taux de profit se trouve donc aggravée. La rentabilisation du capital a rencontré des limites physique » (Gorz, $1977:$ :49-50).

31 En ce contexte, il est important de souligner que non seulement Gorz, mais la plus grande partie des secteurs sociaux partageaient le sentiment que la poursuite du mécanisme capitaliste tel qu'il était jusque-là était impensable.

eles réagissent aux révélations du Club de Rome de deux façons: d'une part, effrayées par un avenir écologiquement incertain mais incapable de ralentir le rythme de croissance - la guerre froide bat son plein - elles investissent beaucoup pour adapter leurs sociétés aux risques environnementaux. Comme l'a clairement montré Romain Felli (2016), dans le débat sur le changement climatique le problème de l'adaptation remonte au milieu des années 70 et précède donc celui de la mitigation d'une décennie. D'autre part, les élites ont préparé une politique de classe extraordinairement agressive - ce que l'on appelle aujourd'hui la contre-offensive néolibérale - visant à proposer à nouveau sur une échelle globale des niveaux de polarisation sociale inconnus depuis la première guerre mondiale. Le pacte social-démocrate commençait à craquer.

De leur coté, les mouvements sociaux ont reconnu la portée historique des limites du développement Fordiste et en ont clairement souligné les enjeux. Selon Gorz (1975), le choix doit se faire entre la démocratie des producteurs associés ou l'autoritarisme planétaire, entre le communisme ou l'éco-fascisme. En bref, l'idée que le capitalisme puisse incorporer les limites bio-sphériques dans les processus de valorisation et en faire ainsi non pas une contrainte mais une vecteur de développement n'était pas à l'ordre du jour. 

possible (Meretz, $2017: 130$ ), son actualisation doit être organisée - pas d'isomorphisme entre la structure de la valorisation et la subjectivité des luttes. Mais ce qui m'intéresse est de mettre en évidence les conditions de possibilité qui ont rendues 'pensable' le gouvernement positif de la crise écologique (c'est-à-dire comme une opportunité et non seulement comme une menace) et 'possible' la formation du Consensus de Paris dans la politique mondiale du climat. On peut fondamentalement identifier - avec Gorz - deux conditions de possibilité. D'abord, la défaite particulière des mouvements sociaux de la période 1968-1973. Il ne fait pas de doute qu'il s'agisse d'un échec : l'objectif de remplacer la logique capitaliste de la valeur par une logique plurielle de richesses n'a pas été atteint. $\mathrm{Au}$ lieu de pouvoir ouvrier, féministe et écologiste sur la composition qualitative de la production a eu lieu la réaction - très violente - du capitale : écrasement du travail, démantèlement de l'État-providence, précarisation des existences. Il convient cependant de noter que la défaite des mouvements a été particulière : la poussée des luttes aboutit en fait à un changement significatif de la structure de la valorisation capitaliste, de plus en plus affranchie du temps de travail comme unité de mesure. Une situation sans précédent a émergé où une réalité d'exploitation croissante et de violence de classe généralisée coexiste avec des potentiels d'émancipation significatifs. En d'autres termes, le commun est devenu un véritable mode de production (Vercellone, Brancaccio, Giuliani, Vattimo, 2017).

La deuxième condition est une conséquence de la première et concerne l'élargissement de la base de l'accumulation capitaliste (Fumagalli 2016) qui se produit à partir de la crise du Fordisme et qui a trois faces : centralité de la reproduction sociale, cognitivisation du travail, financiarisation accélérée de l'économie (à partir de l'abandon unilatéral de 
l'étalon-or - gold standard - par les États-Unis en 1971). D'un point de vue écologique, ces processus font ressortir un espace de valorisation dans lequel le travail-connaissance ne s'applique pas à la nature en tant que limite mais la met plutôt en forme en tant qu'élément direct de la création de valeur. C'est l'ascension, à côté du lien valeur-nature 'classique', d'un lien 'nouveau' basé sur l'exploitation de différents types de travail, pas nécessairement salarié - travail cognitif et/ou reproductif, travail absorbé dans les vortex financiers.

\section{Conclusion}

L'économie verte est une expression de ce lien sans précédent entre la valeur et la nature, et elle devrait être étudiée en tant que telle. L'exploitation qu'elle véhicule n'est pas moins violente de ce qui se donne dans la relation salariale. De plus, la puissance du travail qui s'exprime dans ses circuites est manipulée jusqu'à la dissimulation, tout comme c'était le cas à l'origine avec le travail salarié - tel que Marx l'a découvert via la mise en évidence de la plus-value et, avec elle, de la puissance de la force de travail et du hiatus (à tout profit du capitaliste) entre sa valeur et son prix. L'économie verte, donc, incorpore à la fois le potentiel du travail social enfin conscient de la dimension entropique du salaire comme institution, et sa manipulation capitaliste, c'est-à-dire sa reconduction forcée dans la logique de la valeur - marquée par les impératifs de l'accumulation et de la croissance. En d'autres termes, le devenir productif de la reproduction sociale montre, au cœur même des processus de valorisation, un travail potentiellement néguentropique - non seulement dans le domaine de la reproduction, comme suggéré par Ariel Salleh, mais aussi à l'intérieur de la production. Dans ce cas, on peut voire l'économie verte comme une "idéologie raisonnable»: elle catalyse le nouveau pouvoir du travail, mais le force dans les mailles très étroits de la logique de la valeur.

Une fonction non négligeable de l'économie verte est celle d'obscurcir un objectif politique de bon sens absolu et pourtant (ou peut-être précisément pour ça) incompatible avec l'expansion capitaliste: réduire la part du travail salarié productif (fortement entropique) et augmenter celle du travail reproductif (potentiellement néguentropique). Mais bien sûr, le capital cherche en premier lieu la valeur, pas la salubrité de l'environnement, ni la solidarité entre les (re)producteurs. Sergio Bologna l'avait déjà entrevu avec une clairvoyance extraordinaire dans un article paru en 1987 dans la revue Primo Maggio :

Cette nouvelle révolution industrielle est maintenant sous nos yeux, entièrement déployée : les marchés définis comme 'verts' (en particulier les marchés des émissions et les systèmes de paiement pour les services éco-systémiques [PES]), l'économie numérique et la préservation environnementale apparaît comme une seule totalité financiarisée. La nature mise en valeur dans ces marchés est essentiellement de l'information, c'est-à-dire un mélange d'environnement et de general intellect. Il ne s'agit pas de l'arbre ou de l'océan ou du sol, mais de leur capacité à absorber le dioxyde de carbone par rapport aux besoins des marchés financiers; pas de la graine mais de la séquence génétique qui la rend résistante à un pesticide spécifique produit par l'industrie bio-technologique (Leonardi 2017c).

41 Il apparaît donc un saut d'abstraction dans le lien valeur-nature contemporain, qui cependant ne nie pas le lien 'classique', mais agit plutôt comme un supplément. En fait, 
l'économie vertes reconfigure le lien entre environnement et capital sans le résoudre définitivement. Comme l'a bien montré Daniel Bensaïd (2007), l'incommensurabilité entre la 'valeur' en termes marchands et la 'valeur' en termes écologiques constitue l'une des limites auxquelles le développement historique du mode de production capitaliste est encore soumis.

\section{BIBLIOGRAPHIE}

Bensaïd D, 2007, Les Dépossédés, Paris : La Fabrique.

Bologna S., 1987-1988, « Emarginazione e ambientalismo », Primo Maggio, 27-28, p. 36-41.

Felli R., 2016, La grande adaptation, Paris : Seuil.

Fumagalli A., 2016, La vie mise au travail, Paris : Eterotopia.

Gianinazzi W., 2016, André Gorz : une vie, Paris : La Découverte.

Gollain F., 2018, André Gorz : une philosophie de l'émancipation, Paris : L'Harmattan.

Gorz A., 1967, Le socialisme difficile, Paris : Seuil.

Gorz A., 1972a, «Les impasses de la croissance », in ID., 1973, Critique du capitalisme quotidien, Paris : Galilée, p. 287-297.

Gorz A., 1972b, «Écologie et capitalisme », in ID., 1973, Critique du capitalisme quotidien, Paris : Galilée, p. 298-303.

Gorz A., 1975, Écologie et politique, Paris : Galilée.

Gorz A., 1977, Écologie et liberté, Paris : Galilée.

Gorz A., 1978, « Introduzione all'edizione italiana », in ID., Ecologia e politica, Bologna : Cappelli, p. 8-11.

Gorz A., 2005, « Richesse sans valeur, valeur sans richesse », in ID., 2008, Écologica, Paris : Galilée, p. 65-83.

Gorz A., 2007, « La sortie du capitalisme à déjà commencé », in ID., 2008, Écologica, Paris : Galilée, p. 13-22.

Lavignotte S., 2017, « Première théorisation de l'écologie politique en France », Ecorev' - André Gorz, une pensée vivante, 45, p. 51-56.

Lebert D., Vercellone C., 2004, « L'économie de la connaissance et de l'immatériel, entre théorie et histoire : du capitalisme industriel au capitalisme cognitif », Cahiers lillois d'économie et de sociologie, p. 17-39.

Leonardi E., 2017a, Lavoro Natura Valore : André Gorz tra marxismo e dcrescita, Napoli-Salerno : Orthotes.

Leonardi E., 2017b, «For a Critique of Neoliberal Green Economy : A Foucauldian Perspective on Ecological Crisis and Biomimicry », Soft Power, vol. 5, n 1, p. 168-185. 
Leonardi E., 2017c, « Carbon Trading Dogma », Ephemera, vol. 17, n 1, p. 61-87.

McMahon, J., 2015, « John Kerry Calls For Climate Agreement with 'Legally Binding Transparency System' », Forbes. http://www.forbes.com/sites/jeffmcmahon/2015/12/09/john-kerry-cop-21paris-climate-conference-legally-binding-agreement/. [2015]

Meretz S., 2017, « Vers un communisme de la connaissance ? Dernièrs écrits d'André Gorz », Ecorev' - André Gorz, une pensée vivante, 45, p. 130-138.

Negri A., 2004, « Descartes politico : biopolitica e metafisica », Scienza e Politica, n 31, p. 21-37.

Negri A., 2007, Descartes politico [1970], Roma : Manifestolibri.

Salleh A., 2010, « From Metabolic Rift to 'Metabolic Value' : reflections on Environmental Sociology and the Alternative Globalization Movement », Organization \& Environment, vol. 23, n - 2, p. 205-219.

Vercellone C., Brancaccio F., Giuliani A., Vattimo P., 2017, Il Comune come modo di produzione. Per una critica dell'economia politica dei beni comuni, Verona : Ombre Corte.

\section{NOTES}

1. Cette recherche est cofinancée par le Fonds Social Européen et par les fonds nationaux portugais à travers la Fondation pour la Science et la Technologie dans le cadre de la bourse postdoctorat SFRH / BPD / 96008/2013; Centre d'études sociales, Université de Coimbra. Je remercie Marie Blanc pour l'aide linguistique - très apprécié - avec la rédaction de cet écrit et Françoise Gollain pour m'avoir envoyé certaines citations de Gorz prises des éditions originelles en français.

2. Negri élabore ce concept en 1970, en relation avec la pensée de Descartes: « Il s'agit pour lui, d'une part, de confirmer, du point de vue métaphysique, le pouvoir de la bourgeoisie montante, le potentiel révolutionnaire de son action, la décision de l'autonomie de la raison bourgeoise [...] Si la bourgeoisie se présentait comme une classe hégémonique, capable de construire une nouvelle civilisation, était parce qu'il avait reconnu, à la base de cela, une nouvelle force productive - celle du travail [...] La philosophie de Descartes se lit dans cette clef: en tant qu'idéologie (idéologie dans son sens propre, représentation 'partielle' de la réalité, c'est-à-dire affirmation de la vérité de classe de la bourgeoisie hégémonique) et comme une idéologie raisonnable, planté dans la conscience des relations de pouvoir actuelles et des possibilités progressives, éventuellement ouvert à ce nouveau corps social et à cette vérité » (Negri, 2004 : 25).

\section{INDEX}

Mots-clés : écologie politique, économie verte, general intellect, choc pétrolier de 1973, lien valeur-nature 


\section{AUTEUR}

\section{EMANUELE LEONARDI}

Emanuele Leonardi est post-doctorant au Centro de Estudos Sociais de Universidade de Coimbra (Portugal). 